Generation of broad XUV continuous high harmonic spectra and isolated attosecond pulses with intense mid-infrared lasers

This article has been downloaded from IOPscience. Please scroll down to see the full text article.

2012 J. Phys. B: At. Mol. Opt. Phys. 45011001

(http://iopscience.iop.org/0953-4075/45/1/011001)

View the table of contents for this issue, or go to the journal homepage for more

Download details:

IP Address: 132.246.118.152

The article was downloaded on 16/01/2012 at 20:56

Please note that terms and conditions apply. 


\title{
Generation of broad XUV continuous high harmonic spectra and isolated attosecond pulses with intense mid-infrared lasers
}

\author{
C Trallero-Herrero ${ }^{1,2}$, Cheng Jin ${ }^{1}$, B E Schmidt ${ }^{3}$, A D Shiner ${ }^{2}$, \\ J-C Kieffer ${ }^{3}$, P B Corkum ${ }^{2}$, D M Villeneuve ${ }^{2}$, C D Lin ${ }^{1}$, F Légaré ${ }^{3}$ \\ and $\mathrm{A}$ T Le ${ }^{1}$ \\ ${ }^{1}$ J R Macdonald Laboratory, Physics Department, Kansas State University, Manhattan, KS 66506, USA \\ 2 Joint Attosecond Science Laboratory, National Research Council of Canada and University of Ottawa, \\ Ottawa, ON K1A 0R6, Canada \\ ${ }^{3}$ Institut National de la Recherche Scientifique, Varennes, Québec J3X 1S2, Canada \\ E-mail: trallero@phys.ksu.edu
}

Received 5 October 2011, in final form 5 November 2011

Published 9 December 2011

Online at stacks.iop.org/JPhysB/45/011001

\begin{abstract}
We present experimental results showing the appearance of a near-continuum in the high-order harmonic generation spectra of atomic and molecular species as the driving laser intensity of a mid-infrared pulse increases. Detailed macroscopic simulations reveal that these near-continuum spectra are capable of producing isolated attosecond pulses (IAPs) in the far field if a proper spatial filter is applied. Further, our simulations show that the near-continuum spectra and the IAPs are a product of the strong temporal and spatial reshaping (blue shift and defocusing) of the driving field. This offers a possibility of producing IAPs with a broad range of photon energy, including plateau harmonics, by mid-IR laser pulses even without carrier-envelope phase stabilization.
\end{abstract}

(Some figures in this article are in colour only in the electronic version)

It has been a decade since the first reported generation of IAPs [1] and the first full characterization of an attosecond pulse train (APT) [2], both seminal results that gave birth to the field of attosecond science [3-7]. While APTs can now be routinely generated based on high-order harmonic generation (HHG) in gases, production of IAPs is still a very active field of research. Common to all methods of generating IAPs is the concept of temporal gating, which is used to select a XUV burst from an APT. Current methods can be divided into three different categories. The most intuitive, but technically demanding, approach is based on XUV spectral filtering near the cutoff of the HHG spectra. This approach requires a short driving pulse (about two optical cycles) with a stabilized carrier-envelope phase $[6,8]$. This approach could be called intensity gating, as it selects the harmonics emitted near the peak of the most intense half-cycle of the (short) laser pulse. The second approach is based on polarization gating [9-11], and the generalized double optical gating [12-14], in which the short driving pulse requirement is somewhat relaxed. While these two approaches have their origin already in the single-atom response, the third approach is based on the macroscopic propagation effects [15], which include, ionization gating [16-18], phase matching [19] and ionization-driven reshaping (Gaarde et al $[15,20]$ ). It has also recently been demonstrated that near-continuum spectra can be generated with two-colour multicycle driving laser pulses by carefully adjusting the wavelength of the supplementary pulse [21].

In order to produce a short IAP, XUV radiation with a broad frequency bandwidth needs to be generated. Since the HHG cutoff law is $\Omega_{\text {cutoff }} \sim I_{L} \lambda_{L}^{2}$, there are only two ways to extend $\Omega_{\text {cutoff }}$ : increasing the laser intensity $I_{L}$ or 
increasing the laser wavelength $\lambda_{L}$. The first option is limited by ionization, which dramatically reduces HHG yield due to the depletion of neutral atoms and, more importantly, the phase mismatching due to free electrons in the medium. Using a short laser pulse does not help much, since the current technology nearly reaches the one-cycle limit already. The second option is to use lasers with a long wavelength. This comes with an additional advantage of generating a shorter IAP since the atto-chirp is inversely proportional to the wavelength of the driving laser [22]. The price to pay is that HHG yields have been shown to drastically decrease with wavelength [22-25]. However, it has recently been demonstrated that this decreasing yield with laser wavelength at the single-atom level can be compensated for by increasing the density of atoms resulting in increased XUV flux for some phase matching conditions [26].

In this communication, we present experimental evidence of generation of a broad near-continuum in HHG spectra from atomic and molecular gases by using two-cycle mid-IR $(1825 \mathrm{~nm})$ laser pulses from atomic and molecular targets. Since a continuum spectral emission is consistent with an IAP, we carry out detailed theoretical simulations to show that this scheme is indeed capable of producing IAPs if a proper spatial filter is applied. Our analysis shows that the generation of a near-continuum and an IAP is the result of the combined effect of reshaping the driving laser and phase mismatch, caused by the free electrons due to sub-cycle strong field ionization. With this new method and the light source used, it is possible to use a broad range of harmonics, including plateau harmonics, to generate an IAP with a tunable XUV photon energy.

The experimental setup as shown in figure 1 starts with a Ti:Sa $800 \mathrm{~nm}, 35$ femtosecond laser delivering $6 \mathrm{~mJ}$ of energy per pulse, at a repetition rate of $100 \mathrm{~Hz}$. Using the idler beam of a fluorescence-seeded commercial optical parametric amplifier (OPA, HE TOPAS from Light Conversion), the $800 \mathrm{~nm}$ pulses are converted to $1825 \mathrm{~nm}$. The idler signal from the OPA produces pulses with $52 \mathrm{fs}$ duration and $900 \mu \mathrm{J}$ which are then focused into a $400 \mu \mathrm{m}$ hollow core fibre. Spectral broadening occurs in the capillary due to self-phase modulation and the pulses are compressed by linear propagation through bulk material [27]. The pulse duration measured with a second harmonic generation frequency resolved optical gating device yields $14 \mathrm{fs}$, corresponding roughly to a two-cycle pulse. Following the fibre, the beam is collimated with a $1 \mathrm{~m}$ focal length curved mirror and then focused in a thin $(500 \mu \mathrm{m})$ pulsed jet of atoms or molecules. Before entering the chamber, the beam passes through a half-wave plate and a nanoparticle thin-film polarizer (Thorlabs LPMIR) that allows for intensity control while keeping all other optical parameters constant. Intensity calibration is done using the method outlined in [23]. In short, we measure the ions in parallel with the harmonic emission as a function of laser energy and then fitted to a space and time-averaged Yudin-Ivanov ionization model [28] in a cylindrical geometry [29]. The resulting equations are only intensity dependent once the pulse duration and wavelength are known. All other pulse and detector parameters enter as a single scaling coefficient. Our XUV spectrometer uses a 1200 lines $\mathrm{mm}^{-1}$ ruled grating (Hitachi), which disperses

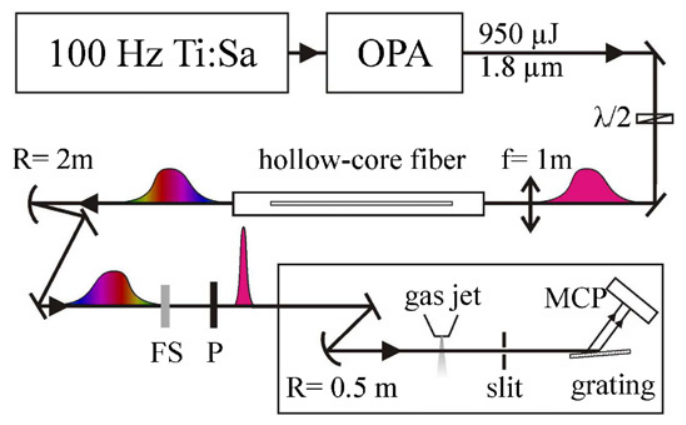

Figure 1. Experimental setup as described in the text.

the light into a microchannel plate/phosphor screen detector (Burle APD 3115 32/25/8 I EDR MgF2 P20). An estimate of the spectrometer resolution can be made by looking at the recombination lines of ionized $\mathrm{O}_{2}$ using circularly polarized light [30]. Through this method, we estimate a spectral resolution of $\leqslant 1 \mathrm{eV}$ in the range of $30-70 \mathrm{eV}$.

In figure 2, we show the HHG spectra obtained in atomic $\mathrm{Xe}$ and molecular NO at different laser intensities. The most striking feature is that the harmonic spectrum becomes a near-continuum as laser intensity increases. This effect is particularly strong for low photon energies $(\Omega<40 \mathrm{eV})$. These continuous spectra extend over a broad range of photon energy from the cutoff at about $100 \mathrm{eV}$ down to very low energy of 20-30 eV. We also observe this feature in other atomic targets as well as in other molecules such as $\mathrm{N}_{2}$, $\mathrm{O}_{2}, \mathrm{CO}, \mathrm{CO}_{2}$ and other organic molecules with relatively low ionization potentials. We therefore believe that this behaviour is quite universal and is independent of atomic and/or molecular species. We also note a saturation effect in observed HHG spectra, when a further increase of laser intensity does not change the HHG spectrum significantly. This can be clearly seen in the case of Xe for laser intensities above about $2 \times 10^{14} \mathrm{~W} \mathrm{~cm}^{-2}$ in figure 2(a).

As the appearance of a broad continuum is an indication of a possible IAP, it is very tempting to speculate this transition as a transition from an APT to IAP by controlling the intensity of few-cycle mid-IR pulses. The transition from APT to IAP at very high laser intensities has been demonstrated previously where the mechanism was ionization gating $[17,31]$. To confirm our hypothesis of the transition from APT to IAP and to further understand the underlying physics, we carried out theoretical simulations, which include the macroscopic propagation of both the driving laser and HHG fields, see below. Our simulations reveal that the mechanism behind this transition is quite different from the conventional ionization gating. In fact, the mechanism is closer to the ionization-driven reshaping of the driving laser pulse, as discussed by Gaarde et al [15, 20]. However, the use of the mid-IR laser pulse leads to a different regime, where the gas pressure is much lower, and the gas jet is much thinner, as compared to that of Gaarde et al $[15,20]$.

Before presenting results of our simulation, we first comment on the differences between the experimental conditions for this work and for our earlier results [32]. The conditions for [32] were chosen to avoid phase mismatch so 


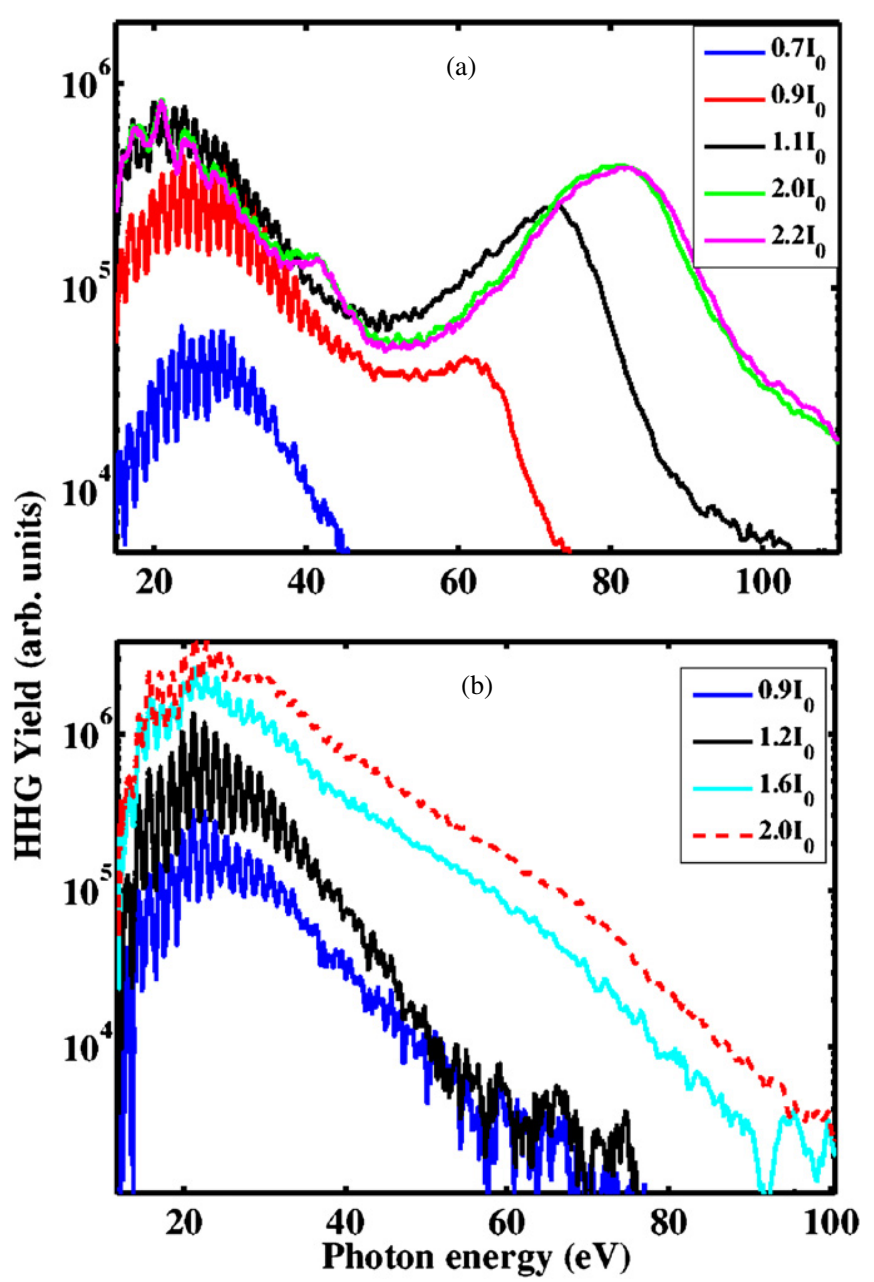

Figure 2. Experimentally measured HHG spectra in Xe (a) and NO (b) for different laser intensities. The driving field has $\lambda_{L}=$ $1825 \mathrm{~nm}$ and duration of $14 \mathrm{fs}$. The intensity values are in units of $I_{0}=10^{14} \mathrm{~W} \mathrm{~cm}^{-2}$.

as to measure harmonic spectra that come as close as possible to the single-atom response. To achieve this, the laser was focused $1-2 \mathrm{~mm}$ downstream from the jet where the gas density was relatively low supporting harmonics which were well phase matched across the entire XUV spectrum. In doing so a broad enhancement in $\mathrm{HHG}$ yield was observed near $100 \mathrm{eV}$, which is attributed to the effect of the inter-channel coupling in the photoionization/photo-recombination cross section of Xe [32]. In the present work, the focus was brought closer to the jet into a region where the gas density was higher. This explains why the cutoff observed after macroscopic propagation falls short of the cutoff expected for the single-atom response. The laser setup used for this work is identical to that in [32] although the pulse duration is somewhat longer (14 fs versus $11 \mathrm{fs})$. The small bump near $40 \mathrm{eV}$ for the highest intensity of $2.0 \times 10^{14} \mathrm{~W} \mathrm{~cm}^{-2}$ in figure 2(a) is an artefact of the grating used for this study. To explain the spectrum that we observe in this work, we performed simulations in atomic Xe at different focusing positions, gas pressure and pulse durations. We found that the most influential parameters are the pressure and the size of the gas jet.
For an in-depth detail of the theoretical method used in this communication, we point the reader towards [33] since we describe only briefly the method here. While in [33] we focus on the role of the CEP in the control of the attosecond pulses, this communication focuses on explaining the transition to a supercontinuum in the harmonic spectrum. The theoretical study of HHG consists of two parts [34-36]. The first one is to calculate the induced dipole of each atom or molecule in the laser field. The second part is to consider the nonlinear propagation of the fundamental laser pulse and the harmonic fields in the medium by solving Maxwell's equations. For the propagation of the fundamental laser field, we include the effects of refraction, nonlinear Kerr effect, ionization and plasma defocusing [35]. Due to the absence of such parameters for most molecules, our simulations focus mainly on atomic Xe. We use the recently developed quantitative rescattering (QRS) theory [37-39] to calculate the induced dipole of a single atom or molecule. Once the induced dipoles are calculated for different laser intensities in the medium, they are then fed into the propagation equations of the harmonic field. At the exit face of the medium, we obtain the near-field harmonic emission. Harmonics then propagate further in the free space, and they may go through a slit before they are collected by the detector. The far-field harmonic emission can be obtained from the near-field harmonic emissions through a Hankel transformation. We pay particular attention to the multi-electron correlated process that occurs in atomic Xe for returning electrons with energies beyond $90 \mathrm{eV}$. The QRS states that the single-atom response induced dipole $D(\omega)$ can be written as

$$
D(\omega)=W(\omega) d(\omega),
$$

with $W(\omega)$ the laser-driven macroscopic wave packet and $d(\omega)$ the photo-recombination (PR) dipole moment. To describe the PR process of $\mathrm{Xe}$, we need to use the PR transition dipole moment including multielectron effects in QRS theory. Such a PR moment can be extracted from the experimental photoionization cross section (PICS) [40, 41]. In the calculation, we incorporate the PICS of Xe, which is extended into the high-photon energy region (a few hundred $\mathrm{eV}$ ), using the relativistic random-phase approximation (RRPA) by Kutzner et al [42]. Note that the phase of PR transition dipole moment is assumed to be the same as that solely caused by $5 \mathrm{p}$.

The results of our simulations in atomic Xe are shown in figure 3. First, the CEP-averaged HHG spectra of the singleatom response obtained with the QRS method are shown in figure 3(a) for two laser intensities of $0.5 \times 10^{14}$ and $1.0 \times 10^{14} \mathrm{~W} \mathrm{~cm}^{-2}$. Clearly, the simulation at the singleatom level does not reproduce the transition to the continuum spectrum, observed in the experiment, as well as the HHG cutoff position for the high laser intensity. The ionization probability is about $10-30 \%$ at the end of the pulse in this range of laser intensity. This result basically excludes the possibility of ionization gating at the single-atom level, as a mechanism for the transition to the near-continuum spectra. We also found that a further increase in the laser intensity up to $2.0 \times 10^{14} \mathrm{~W} \mathrm{~cm}^{-2}$ does not change this behaviour. We comment that the generation of an IAP with a high pulse 


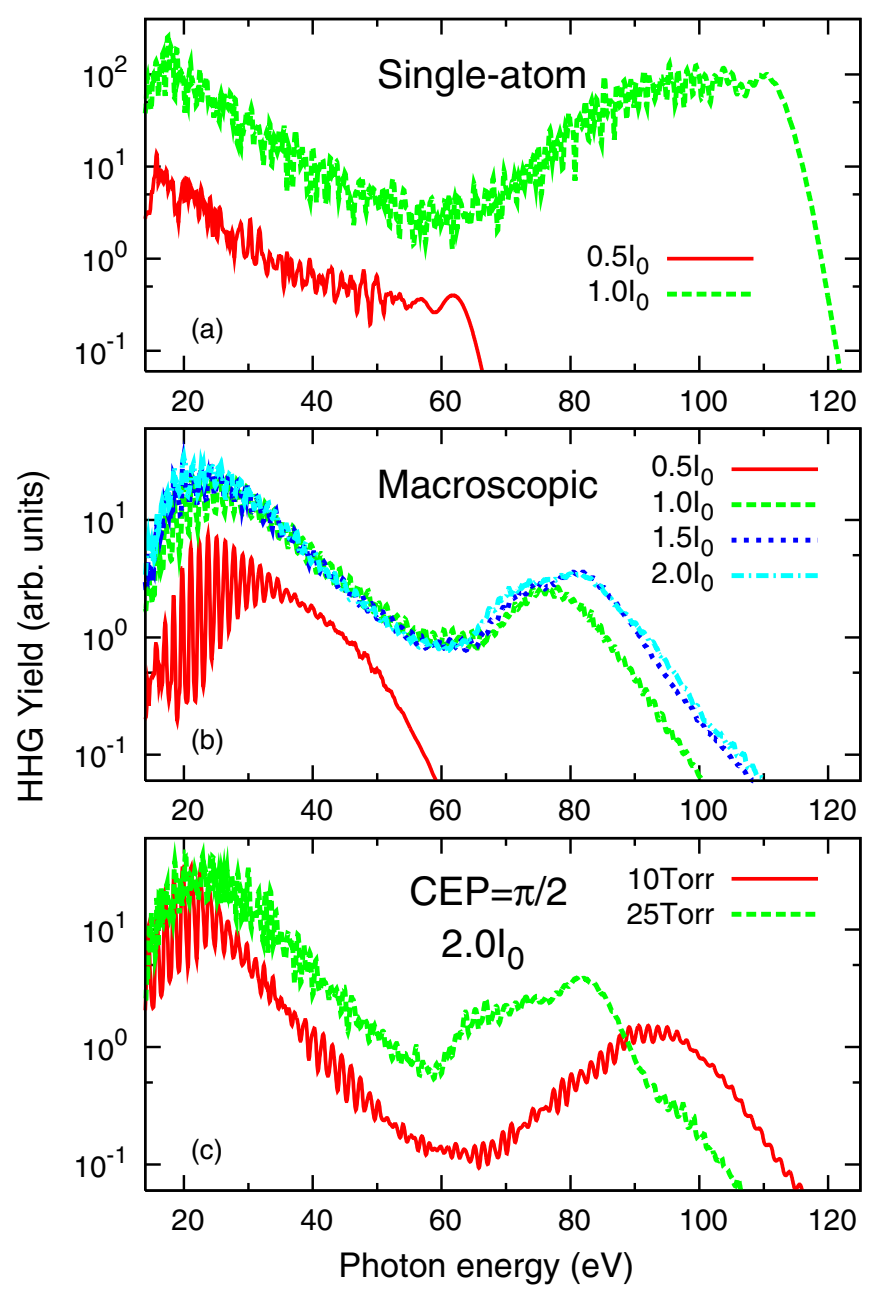

Figure 3. HHG spectra calculated using QRS theory for different peak intensities as indicated where $I_{0}=10^{14} \mathrm{~W} \mathrm{~cm}^{-2}$. (a) Spectra for a single-atom response. (b) CEP-averaged spectra from macroscopic simulations for different intensities. (c) Spectra from macroscopic simulations for gas pressures of 10 and 25 torr, at a $\mathrm{CEP}$ of $\pi / 2$. Other laser parameters are the same as in the experiment for all panels.

energy (few nanojoules) has recently been reported by Ferrari et al [31], which is based on complete depletion of the neutral $\mathrm{Xe}$ atoms.

Next, we show in figure 3(b) the harmonic spectra for four peak laser intensities after propagation through the gas jet with parameters that closely resemble the experimental conditions. Such a condition includes not only the laser parameters but also the jet size $(1 \mathrm{~mm})$, the spectrometer slit opening $(190 \mu \mathrm{m})$ and distance of the slit to the interacting region $(455 \mathrm{~mm})$. In the theoretical calculations, the beam is assumed to be Gaussian. All spectra are averaged over random values of the CEP as in the experiment. The macroscopic simulations indeed show a transition to a near-continuum HHG spectrum for a broad range of photon energy from the 'apparent' cutoff down to about $30 \mathrm{eV}$, as the laser intensity increases, thus reproducing nicely the experimental finding. The simulations also reproduce the saturation effect observed in the experiments. This is not the case for the single-atom simulations shown in figure 3 (a). This leads us to conclude that the near-continuum is a consequence of the propagation in the medium of both the fundamental and the harmonics fields.

By comparing HHG spectra obtained for the single atom with the ones from a macroscopic simulation for the same peak intensities, we observe large discrepancies in the cutoff and the overall shape. Before explaining these discrepancies as well as the physical mechanism behind the experimentally observed spectra, we address here the differences between the current experimental data and that of Shiner et al [32]. Figure 3(c) shows a comparison between the HHG spectra from macroscopic simulations for two different gas pressures of 10 and 25 torr. The laser intensity is $2.0 \times 10^{14} \mathrm{~W} \mathrm{~cm}^{-2}$ and the CEP is chosen to be $\pi / 2$. The 'apparent' cutoffs are different by about $15 \mathrm{eV}$. Thus, the discrepancy between the two experiments can be tentatively attributed as due to the different gas pressures. Moreover, we note that the size of the gas jet could also affect the cutoff position and the overall shape of HHG spectra. A thinner gas jet would give a spectrum closer to a single-atom response, i.e. with a higher cutoff.

In order to understand the mechanism behind the transition to the near-continuum HHG spectrum, we followed the fundamental field in space and time through the simulation. In figure 4, we show the on-axis electric field amplitude as a function of time at the entrance, middle and just before the exit of the Xe gas jet, for gas pressures of 25 and 10 torr. The CEP is chosen to be $\pi / 2$, and the laser intensity is $2.0 \times 10^{14} \mathrm{~W} \mathrm{~cm}^{-2}$. As can be seen from the figure, the temporal shape of the laser pulse is strongly modified as it propagates though the medium. In particular, a dynamic blue shift (or chirp) can also be seen clearly. In fact, this shape is quite typical for the self-phase modulation found in a rapidly ionizing medium $[15,20]$. A chirped pulse means that harmonics will be produced at different fundamental frequencies depending on where and when they are generated. The effect of chirp in HHG has already been established [43, 44] and it induces a frequency shift in the observed harmonic spectrum. In our case, the harmonics are produced with different chirped pulses all at once. Furthermore, the electron density strongly varies as a function of radial distance due to the radial variation of the laser intensity. This radial variation electron density acts like a negative lens to defocus the laser beam. We found that both temporal and spatial reshaping are quite negligible at the laser intensity of $0.5 \times 10^{14} \mathrm{~W} \mathrm{~cm}^{-2}$, since the ionization is insignificant. This explains the observed transition to the near-continuum in HHG spectra as the laser intensity increases.

The temporal and spatial reshaping of the laser beam significantly reduce the laser intensity in the medium, which accounts for the 'apparent' lower energy cutoff in the HHG spectra found in the macroscopic simulations, as compared to the single-atom simulations. A further increase in the laser intensity above $2.0 \times 10^{14} \mathrm{~W} \mathrm{~cm}^{-2}$ will lead to even the stronger reshaping and redistribution of laser intensity, which explains the saturation effect discussed above.

The reshaping of the laser pulse has been discussed before in the context of the $800 \mathrm{~nm}$ driving pulse $[15,20]$. The effect is even more significant for the mid-IR laser, since the effective index of refraction due to free electron is $\eta_{e} \sim-n_{e} \lambda_{L}^{2}$, where 


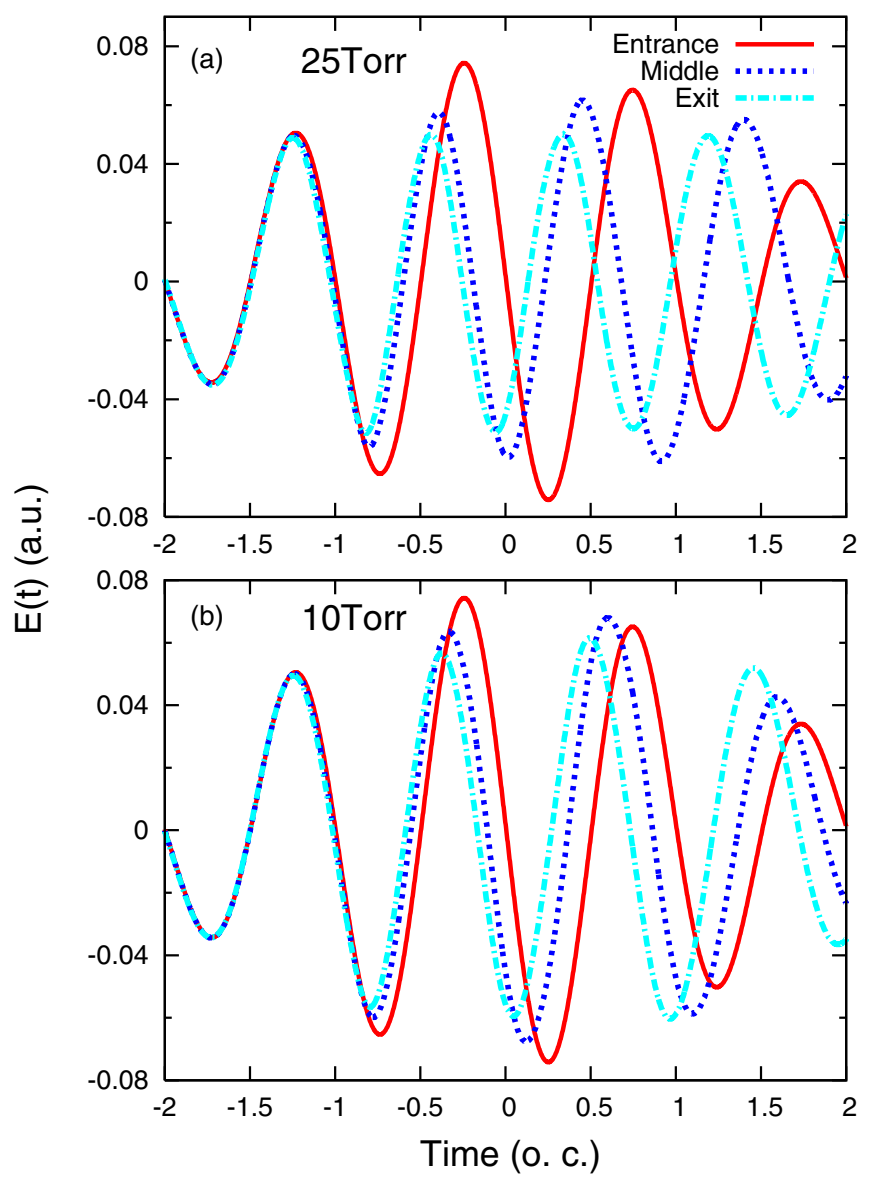

Figure 4. Driving electric field $\left(\lambda_{L}=1825 \mathrm{~nm}\right)$ as a function of time (in units of the optical cycle) at three different positions relative to the atomic jet, for gas pressures of 25 (a) and 10 torr (b). All values are 'measured' on axis. The laser parameters are $I_{L}=2.0 \times 10^{14} \mathrm{~W} \mathrm{~cm}^{-2}$ and $\mathrm{CEP}=\pi / 2$.

$n_{e}$ is the density of free electron and $\lambda_{L}$ is the wavelength of the driving laser. Therefore, the reshaping of the laser pulse occurs at much lower gas pressure and size (25 torr, $1 \mathrm{~mm}$ long) as compared to that of Gaarde et al $[15,20]$ (135 torr, $3 \mathrm{~mm}$ long). We further comment that at the lower pressure of 10 torr, shown in figure 4(b), the reshaping is less severe as compared to that at 25 torr, thus leading to the higher HHG cutoff seen in figure 3(c).

To further understand the nature of the near-continuum and to address the usefulness of our scheme for the production of IAPs, we follow the attosecond pulses in time as they emerge from the interacting region. We use a spectral filter to select harmonics from H40 (27.2 eV) to H80 (54.4 eV). Figure 5(a) shows the attosecond pulses using these harmonics for the same driving field $\left(\lambda_{L}=1825 \mathrm{~nm}\right.$ and duration (FWHM) of $14 \mathrm{fs})$ with a peak intensity of $1.5 \times 10^{14} \mathrm{~W} \mathrm{~cm}^{-2}$, and a CEP of $\pi / 2$ at the end of the interacting region (near field). The presence of an APT is evident. As proposed in [15], an IAP can be obtained in the far field by spatially filtering the XUV pulses. In fact, due to the temporal and spatial reshaping, the HHG emission at different times will have a different divergence. Figure 5(b) shows harmonics H40 to H80 in time domain, under the same conditions as panel (a), but the pulse is 'measured' in the far field after a circular filter

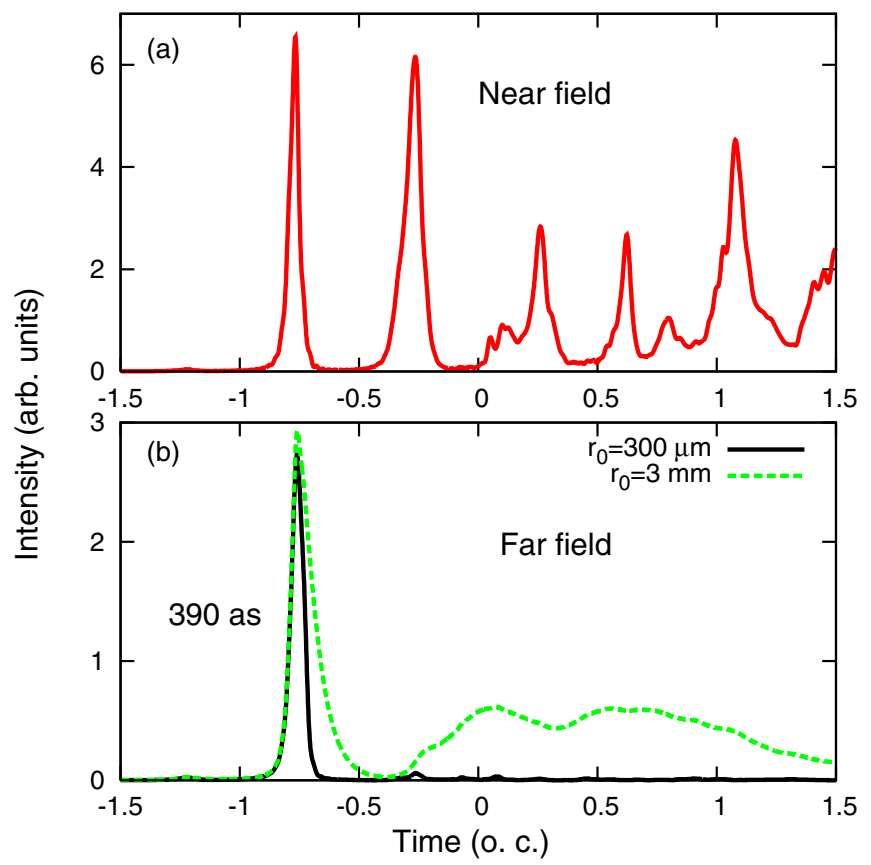

Figure 5. (a) Intensity of attosecond pulses in the near field.

(b) Intensity of IAP in the far field (measured $455 \mathrm{~mm}$ after the laser focus). Black denotes that a spatial filter with a radius $r_{o}=300 \mu \mathrm{m}$, located $455 \mathrm{~mm}$ after the focus, is used. Green denotes that a spatial filter of $r_{o}=3 \mathrm{~mm}$, at the same position, is used instead. Harmonics used to synthesize attosecond pulses are $\mathrm{H} 40(27.2 \mathrm{eV})$ to $\mathrm{H} 80$ $(54.4 \mathrm{eV})$. The laser intensity at the focus is $1.5 \times 10^{14} \mathrm{~W} \mathrm{~cm}^{-2}$ and the CEP is $\pi / 2$.

with a radius of $300 \mu \mathrm{m}$, located $455 \mathrm{~mm}$ after the focus. In this case, a single 390 as pulse is indeed 'measured', thus proving that the observed XUV supercontinuum can indeed produce IAPs. This attosecond burst is generated in the leading edge of the pulse, less than one optical cycle before the peak intensity is reached. Clearly, the more divergent attosecond bursts, generated at later times, have been blocked by the filter. This result also indicates that the phase mismatching due to the free electron might also play an important role, as the nearaxis contribution from the later sub-cycles is suppressed due to the phase mismatch. We mention that the phase mismatch due to the free electron is more severe for the long wavelength. Figure 5(b) also shows in green the XUV pulse under the same conditions but using a spatial filter with a radius of $3 \mathrm{~mm}$. In this case, an IAP is not obtained, thus showing the importance of the filtering. We also carried out simulations with a lower laser intensity of $0.5 \times 10^{14} \mathrm{~W} \mathrm{~cm}^{-2}$. The result shows an APT even after the spatial filter is applied. At this low intensity, the ionization and the pulse reshaping are insignificant. Therefore, in this case different XUV bursts at different half-cycles will have similar divergence.

We emphasize that relatively low harmonics is chosen here to illustrate the usefulness of our scheme for producing an IAP with plateau harmonics. In contrast, in a typical IAP generation with the reshaping mechanism with $800 \mathrm{~nm}$ laser pulses, only the harmonics near the cutoff are used for producing an IAP [15]. Furthermore, such IAPs can be manipulated by changing the CEP of the driving field, as well as the spectral and spatial filters. In particular, these IAPs can 
be produced even without CEP stabilized laser pulses for most of the CEP values [33]. Although the experimental results and theoretical analysis presented here are for two-cycle pulses, we anticipate that production of IAPs within our scheme is also feasible with longer pulses.

In conclusion, we have observed a near continuum in the HHG spectrum, with a $1.8 \mu \mathrm{m}$ laser driver as the laser intensity increases. By performing theoretical simulations of the full macroscopic HHG process combined with a realistic singleatom response calculation, we are able to reproduce the same behaviour as we observe in the experiment. The simulations show that the transition from a train of attosecond pulses to an IAP is due to the strong reshaping of the driving laser as it propagates through the medium. The main advantage of our approach for producing IAPs is that the central frequency of the XUV pulse can be tuned over a broader range of photon energy as compared to the standard $800 \mathrm{~nm}$. This is due to the capability of using a large range of harmonics, including plateau harmonics, of a broad near-continuum spectrum. We comment that our method is based on a different mechanism from that of Ferrari et al [31], which is based on complete depletion of the neutral atoms. However, in both methods, both plateau and cutoff harmonics can be used to synthesize IAPs. Finally, it has recently been reported [26] that the XUV yield can increase with wavelength when proper phase matching conditions are exploited, thus creating a great venue for our approach.

\section{Acknowledgments}

This work was supported in part by the Chemical Sciences, Geosciences and Biosciences Division, Office of Basic Energy Sciences, Office of Science, US Department of Energy. CT-H also thanks the AFOSR for their financial support.

\section{References}

[1] Hentschel M, Kienberger R, Spielmann C, Reider G A, Milosevic N, Brabec T, Corkum P, Heinzmann U, Drescher M and Krausz F 2001 Nature 414509

[2] Paul P M, Toma E S, Breger P, Mullot G, Augé F, Balcou P, Muller H G and Agostini P 2001 Science 2921689

[3] Corkum P B and Krausz F 2007 Nature Phys. 3381

[4] Krausz F and Ivanov M 2009 Rev. Mod. Phys. 81163

[5] Corkum P B and Chang Z 2008 Opt. Photon. News 1924

[6] Christov I P, Murnane M M and Kapteyn H C 1997 Phys. Rev. Lett. 781251

[7] Popmintchev T, Chen M C, Arpin P, Murnane M M and Kapteyn H C 2010 Nature Photon. 4822

[8] Goulielmakis E et al 2008 Science 3201614

[9] Corkum P B, Burnett N H and Ivanov M Y 1994 Opt. Lett. 191870

[10] Tcherbakoff O, Mével E, Descamps D, Plumridge J and Constant E 2003 Phys. Rev. A 68043804

[11] Sola I J et al 2006 Nature Phys. 2319

[12] Mashiko H, Gilbertson S, Li C, Khan S D, Shakya M M, Moon E and Chang Z 2008 Phys. Rev. Lett. 100103906
[13] Feng X, Gilbertson S, Mashiko H, Wang H, Khan S D, Chini M, Wu Y, Zhao K and Chang Z 2009 Phys. Rev. Lett. 103183901

[14] Gilbertson S, Khan S D, Wu Y, Chini M and Chang Z 2010 Phys. Rev. Lett. 105093902

[15] Gaarde M B, Tate J L and Schafer K J 2008 J. Phys. B: At. Mol. Opt. Phys. 41132001

[16] Pfeifer T, Jullien A, Abel M J, Nagel P M, Gallmann L, Neumark D M and Leone S R 2007 Opt. Express 1517120

[17] Abel M J, Pfeifer T, Nagel P M, Boutu W, Bell M J, Steiner C P, Neumark D M and Leone S R 2009 Chem. Phys. 3669

[18] Thomann I, Bahabad A, Liu X, Trebino R, Murnane M M and Kapteyn H C 2009 Opt. Express 174611

[19] Haworth C A, Chipperfield L E, Robinson J S, Knight P L, Marangos J P and Tisch J W G 2007 Nature Phys. 352

[20] Gaarde M B and Schafer K J 2006 Opt. Lett. 313188

[21] Takahashi E J, Lan P, Mücke O D, Nabekawa Y and Midorikawa K 2010 Phys. Rev. Lett. 104233901

[22] Tate J, Auguste T, Muller H G, Salières P, Agostini P and DiMauro L F 2007 Phys. Rev. Lett. 98013901

[23] Shiner A D, Trallero-Herrero C, Kajumba N, Bandulet H-C, Comtois D, Légaré F, Kieffer J-C, Corkum P B and Villeneuve D M 2009 Phys. Rev. Lett. 10307390

[24] Frolov M V, Manakov N L and Starace A F 2008 Phys. Rev. Lett. 100173001

[25] Schiessl K, Ishikawa K L, Persson E and Burgdörfer J 2007 Phys. Rev. Lett. 99253903

[26] Popmintchev T, Chen M-C, Bahabad A, Gerrity M, Sidorenko P, Cohen O, Christov I P, Murnane M M and Kapteyn H C 2009 Proc. Natl Acad. Sci. USA 10610516

[27] Schmidt B E et al 2010 Appl. Phys. Lett. 96121109

[28] Yudin G L and Ivanov M Y 2001 Phys. Rev. A 64013409

[29] Hankin S, Villeneuve D, Corkum P and Rayner D 2001 Phys. Rev. A 64013405

[30] Farrell J P, McFarland B K, Bucksbaum P H and Gühr M 2009 Opt. Express 1715134

[31] Ferrari F, Calegari F, Lucchini M, Vozzi C, Stagira S, Sansone G and Nisoli M 2010 Nature Photon. 4875

[32] Shiner A D, Schmidt B E, Trallero-Herrero C, Worner H J, Patchkovskii S, Corkum P B, Kieffer J-C, Legare F and Villeneuve D M 2011 Nature Phys. 7464

[33] Jin C, Le A-T, Trallero-Herrero C A and Lin C D 2011 Phys. Rev. A 84043411

[34] Jin C, Le A-T and Lin C D 2009 Phys. Rev. A 79053413

[35] Jin C, Le A-T and Lin C D 2011 Phys. Rev. A 83023411

[36] Jin C, Wörner H J, Tosa V, Le A-T, Bertrand J B, Lucchese R R, Corkum P B, Villeneuve D M and Lin C D 2011 J. Phys. B: At. Mol. Opt. Phys. 44095601

[37] Lin C D, Le A-T, Chen Z, Morishita T and Lucchese R 2010 J. Phys. B: At. Mol. Opt. Phys. 43122001

[38] Morishita T, Le A-T, Chen Z and Lin C D 2008 Phys. Rev. Lett. 100013903

[39] Le A-T, Lucchese R R, Tonzani S, Morishita T and Lin C D 2009 Phys. Rev. A 80013401

[40] Becker U, Szostak D, Kerkhoff H G, Kupsch M, Langer B, Wehlitz R, Yagishita A and Hayaishi T 1989 Phys. Rev. A 393902

[41] Fahlman A, Krause M O, Carlson T A and Svensson A 1984 Phys. Rev. A 30812

[42] Kutzner M, Radojević V and Kelly H P 1989 Phys. Rev. A 405052

[43] Zhou J, Peatros J, Murnane M M and Kapteyn H C 1996 Phys. Rev. Lett. 76752

[44] Kim H T, Kim I J, Hong K-H, Lee D G, Kim J-H and Nam C H 2004 J. Phys. B: At. Mol. Opt. Phys. 371141 